\title{
Antidepressants: Is a higher dose always better?
}

Aaron N. Tran, PharmD, BCPP, BCPS, and Risa Ishino, PharmD, BCPP, BCPS

$\mathrm{M}$ r. $E$, age 39 , presents to the mental health $(\mathrm{MH})$ intake clinic, reporting he has had depressed mood almost every day, lack of interests, poor appetite, difficulty sleeping, inability to concentrate on daily activities, low energy and motivation, and feelings of guilt. He is diagnosed with major depressive disorder and agrees to a trial of sertraline, which is titrated up to 100 $\mathrm{mg} / \mathrm{d}$. He is also referred to the MH pharmacy clinic for interim visits.

Four weeks later during a follow-up visit, Mr. E reports tolerating sertraline, $100 \mathrm{mg} / \mathrm{d}$, with a slight improvement in his mood. He reports that he has started working on his previous hobbies again and tries to consistently eat 2 meals a day. He feels that his sleep remains unchanged. He would like to enroll in school again, but is concerned about his poor concentration. He asks whether a further increase in his sertraline dose would improve his symptoms. What would you advise?

Escalating antidepressant doses up to, or even above, the FDA-approved maximum dose is a strategy for clinicians to consider for patients who are nonresponders or partial responders to treatment. This practice assumes that the effectiveness of an antidepressant is dependent on the dosage.

Dr. Tran is Clinical Pharmacist Specialist, Mental Health VA Loma Linda Healthcare System, Loma Linda, California. Dr. Ishino is Clinical Pharmacist Specialist, Mental Health VA Loma Linda Healthcare System, Loma Linda, California.

Disclosures

The authors report no financial relationships with any companies whose products are mentioned in this article, or with manufacturers of competing products.

doi: $10.12788 /$ cp.0102
However, based on our review of available literature, this recommendation is equivocally supported for general practice.

\section{Selective serotonin reuptake inhibitors}

The Table Th $^{1-3}$ page 40) summarizes the results of 3 studies of high-dose selective serotonin reuptake inhibitors (SSRIs).

Adli et al ${ }^{1}$ evaluated 3 types of studiesstudies of patients with treatment-resistant depression receiving high-dose treatment, comparative dose studies, and studies of therapeutic drug-monitoring (TDM) of antidepressants-to assess the effectiveness of high-dose antidepressants after a treatment failure with a medium dose. They concluded that SSRIs exhibit a flat dose-dependency pattern, where increasing a dose above the minimum effective dose (MED) does not increase efficacy but results in more adverse effects. Because treatment at the MED

\section{Practice Points}

- Recommending an antidepressant dose escalation assumes a dose-response relationship.

- Compared with other types of antidepressants, tricyclic and tetracyclic antidepressants appear to have more evidence supporting a benefit from dose escalation.

- Current evidence equivocally supports dose escalation for selective serotonin reuptake inhibitors.

- Consider an individual patient's pharmacogenetic and relevant pharmacokinetic factors before initiating a dose escalation.

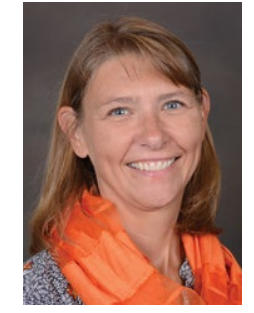

Vicki L. Ellingrod, PharmD, FCCP Department Editor
Savry Psychopharmacology is produced in partnership with the College of Psychiatric and Neurologic Pharmacists cpnp.org mhc.cpnp.org (journal) 


\section{Clinical Point}

Evidence supporting antidepressant dose escalation is inconclusive, but higher doses result in more adverse effects

Discuss this article at www.facebook.com/ MDedgePsychiatry

\section{Table}

\section{Dose escalation of selective serotonin reuptake inhibitors: 3 studies}

\begin{tabular}{|c|c|c|}
\hline Study & Design & Conclusion \\
\hline Adli et al $^{1}$ & $\begin{array}{l}\text { Review of } 3 \text { types of studies: 1) dose } \\
\text { increase studies in treatment-refractory } \\
\text { patients, 2) comparative dose studies, and } \\
\text { 3) therapeutic drug monitoring studies }\end{array}$ & $\begin{array}{l}\text { SSRIs exhibit a flat dose-dependency } \\
\text { pattern, where an increase in dose above } \\
\text { the minimum effective dose does not } \\
\text { increase efficacy but results in more } \\
\text { adverse effects }\end{array}$ \\
\hline Ruhe et $\mathrm{al}^{2}$ & $\begin{array}{l}\text { Review of } 8 \text { randomized controlled trials } \\
\text { and } 3 \text { systematic analyses that examined } \\
\text { dose escalation of SSRIs, including } \\
\text { paroxetine, fluoxetine, and sertraline }\end{array}$ & $\begin{array}{l}\text { Evidence for increased efficacy with } \\
\text { SSRI dose escalation was inconclusive, } \\
\text { but dose escalation resulted in more } \\
\text { adverse effects }\end{array}$ \\
\hline $\begin{array}{l}\text { Hieronymus } \\
\text { et } \mathrm{al}^{3}\end{array}$ & $\begin{array}{l}\text { Mega-analysis of dose-dependency } \\
\text { patterns of selected SSRIs, including } \\
\text { citalopram, paroxetine, and sertraline, in } \\
\text { adult patients with depression }\end{array}$ & $\begin{array}{l}\text { SSRIs at low doses were superior to } \\
\text { placebo but inferior to higher doses. The } \\
\text { dose-response relationship plateaued } \\
\text { at } 20 \mathrm{mg} / \mathrm{d} \text { for paroxetine, } 40 \mathrm{mg} / \mathrm{d} \text { for } \\
\text { citalopram, and } 100 \mathrm{mg} / \mathrm{d} \text { for sertraline }\end{array}$ \\
\hline
\end{tabular}

inhibits $70 \%$ of serotonin reuptake and is only marginally less effective than medium therapeutic doses, the authors recommended reserving treatment at higher doses for patients who have failed other standard treatment options, such as augmentation.

Ruhe et $\mathrm{al}^{2}$ evaluated 8 randomized controlled trials and 3 systematic analyses that investigated dose escalation of SSRIs, including paroxetine, fluoxetine, and sertraline. The authors noted that all included studies had methodological limitations and discussed 1 study that showed potential benefit from dose escalation when dropouts due to adverse effects were excluded from analysis. They determined that the evidence for increased efficacy with dose escalation was inconclusive; however, dose escalation undoubtedly resulted in more adverse effects.

Hieronymus et $\mathrm{al}^{3}$ found a dosedependency pattern with selected SSRIscitalopram, paroxetine, and sertraline-in a mega-analysis of studies of adult patients with depression. All company-funded, acute-phase, placebo-controlled fixed-dose trials of these agents were included in this analysis. It included a total of 2,859 patients: 600 patients received citalopram (10 to $60 \mathrm{mg} / \mathrm{d}) ; 1,043$ patients received paroxetine (10 to $40 \mathrm{mg} / \mathrm{d}$ ); 481 patients received sertraline (50 to $400 \mathrm{mg} / \mathrm{d}$ ); and 735 patients received placebo. They further divided the SSRIs into "low" vs "optimal" doses based on the dose curves of these agents. For citalopram, 10 to $20 \mathrm{mg} / \mathrm{d}$ was considered low vs 40 to $60 \mathrm{mg} / \mathrm{d}$, which was considered optimal. For paroxetine, $10 \mathrm{mg} / \mathrm{d}$ was considered low vs other doses as optimal (20, 30 , and $40 \mathrm{mg} / \mathrm{d}$ ). For sertraline, $50 \mathrm{mg}$ was considered low vs other doses as optimal (100, 200, and $400 \mathrm{mg} / \mathrm{d})$. The authors concluded that at low doses, these antidepressants were superior to placebo but inferior to higher doses. Interestingly, they suggested that the dose-response relationship plateaued at $20 \mathrm{mg} / \mathrm{d}$ for paroxetine, $40 \mathrm{mg} / \mathrm{d}$ for citalopram, and $100 \mathrm{mg} / \mathrm{d}$ for sertraline. One of the limitations of the study was a lack of information on the tolerability of higher vs lower doses.

\section{Other antidepressants}

Adli et $\mathrm{al}^{1}$ found a high-dose study and several comparative studies that supported a dose-response relationship with a reasonable degree of tolerability for venlafaxine, but there were no pertinent studies that evaluated mirtazapine. The only fixed-dose study found for bupropion did not support a dose-response relationship. ${ }^{1}$

The authors also concluded that there may be evidence supporting high-dose 
prescribing of tricyclic and tetracyclic antidepressants (TCAs and TeCAs, respectively). Despite the lack of clinical data that directly addressed the dose-dependency of TCAs and TeCAs, the authors supported dose escalation with amitriptyline, clomipramine, imipramine, desipramine, nortriptyline, and maprotiline, based on the data from comparative dose and TDM studies. ${ }^{1}$ The authors urged caution in interpreting and applying the results of TDM studies because the pharmacodynamic of each medication-such as being linear, curvilinear, or uncorrelatedmay vary, which suggests there is a targeted therapeutic dose range. ${ }^{1}$

\section{Important considerations}

Differences in the pharmacokinetic and pharmacogenetic properties of individual medications may account for the mixed outcomes found when evaluating antidepressant dose-response relationships. Genetic polymorphisms of cytochrome (CYP) P450

\section{Related Resources}

- Berney P. Dose-response relationship of recent antidepressants in the short-term treatment of depression. Dialogues Clin Neurosci. 2005;7:249.

- Jakubovski E, Varigonda AL, Freemantle N, et al. Systematic review and meta-analysis: dose-response relationship of selective serotonin reuptake inhibitors in major depressive disorder. Am J Psychiatry. 2016;173:174-183.

Drug Brand Names

Amitriptyline - Elavil Bupropion - Wellbutrin Citalopram - Celexa Clomipramine - Anafranil Desipramine - Norpramin Fluoxetine - Prozac Imipramine - Tofranil

enzymes, mainly CYP2D6 and CYP2D19, have been shown to directly affect antidepressants' serum levels. Depending on the patient's phenotype expression, such as poor, intermediate, extensive (ie, normal), or ultra-metabolizers, use of a specific
Maprotiline $\cdot$ Ludiomil Mirtazapine $\cdot$ Remeron Nortriptyline $\cdot$ Pamelor Paroxetine $\cdot$ Paxil Sertraline - Zoloft Venlafaxine - Effexor
Clinical Point

Before initiating a dose escalation, consider a patient's pharmacogenetic and relevant pharmacokinetic factors 
antidepressant at a similar dose may result in therapeutic effectiveness, ineffectiveness, or toxicity. For antidepressants such as TCAs, which have a narrow therapeutic index compared with SSRIs, the differences in pharmacokinetic and pharmacogenetic properties becomes more impactful. ${ }^{1,4}$

\section{Escalation within approved dose ranges}

Few quality studies have conclusively found a relationship between antidepressant dose escalation within the FDA-approved dose ranges and efficacy, and there are few to no recommendations for prescribing doses above FDA-approved ranges. However, in clinical practice, clinicians may consider a dose escalation within the allowable dose ranges based on anecdotal evidence from previous patient cases. Consideration of relevant pharmacokinetic parameters and the patient's individual pharmacogenetic factors may further guide clinicians and patients in making an informed decision on dose escalation to and beyond the FDAapproved doses.

\section{CASE CONTINUED}

After reviewing the evidence of antidepressant dose escalation and Mr. E's progress, the $\mathrm{MH}$ pharmacist recommends that the psychiatrist increase Mr. E's sertraline to $150 \mathrm{mg} / \mathrm{d}$ with close monitoring.

\section{References}

1. Adli M, Baethge C, Heinz A, et al. Is dose escalation of antidepressants a rational strategy after a medium-dose treatment has failed? A systematic review. Eur Arch Psychiatry Clin Neurosci. 2005;255(6):387-400.

2. Ruhe HG, Huyser J, Swinkels JA, et al. Dose escalation for insufficient response to standard-dose selective serotonin reuptake inhibitors in major depressive disorder. Bri J Psychiatry. 2006;189:309-316.

3. Hieronymus F, Nilsson S, Eriksson E. A mega-analysis of fixed-dose trials reveals dose dependency and a rapid onset of action for the antidepressant effect of three selective serotonin reuptake inhibitors. Transl Psychiatry. 2016;6(6):e834. doi: 10.1038/tp.2016.104

4. Nassan M, Nicholson WY, Elliott MA, et al. Pharmacokinetic pharmacogenetic prescribing guidelines for antidepressants: a template for psychiatric precision medicine. Mayo Clin Proc. 2016;91(7):897-907. 